\title{
Efficacité des alertes signalant un risque de collision par l'arrière chez le conducteur distrait
}

\author{
Efficiency of alerts indicating a rear-end collision risk in distracted drivers
}

\author{
Alexandra Fort · Mercedes Bueno · Colette Fabrigoule · Jordan Navarro · Daniel Ndiaye · Christophe Jallais \\ (C) IFSTTAR et Éditions NecPlus 2015
}

\begin{abstract}
Résumé Ce papier présente une revue de la littérature visant à apporter un éclairage sur l'impact des distractions sur l'efficacité d'alerte signalant un risque de collision.

Les collisions par l'arrière représentent une part non négligeable des accidents corporels. Un des facteurs contributifs majeurs à ces collisions est l'inattention des conducteurs. Afin de diminuer l'impact de ces collisions, des assistances à la conduite de type alerte ont vu le jour. L'efficacité de ces alertes a été évaluée dans différentes situations (par simulation, sur simulateur et sur route) chez des conducteurs non distraits et distraits. Dans le dernier cas, la majorité des tâches distractives employées impliquent un détournement du regard de la route vers un
\end{abstract}

Alexandra Fort $(\triangle)$

Ifsttar-TS2-LESCOT

25 avenue François Mitterrand, 69675 Bron Cedex, France

e-mail : alexandra.fort@ifsttar.fr

\section{Mercedes Bueno $(\square)$}

Ifsttar-TS2-LESCOT

25 avenue François Mitterrand, 69675 Bron Cedex, France

e-mail : mercedes.bueno-garcia@ifsttar.fr

Colette Fabrigoule $(\square)$

Université Bordeaux Segalen, USR CNRS 3413 SANPSY,

CHU Pellegrin, F-33076 Bordeaux, France

e-mail : colette.fabrigoule@imf.u-bordeaux2.fr

Jordan Navarro $(\bowtie)$

Université Lumière Lyon 2, Laboratoire d'Etude des Mécanismes

Cognitifs (EMC)

5 Avenue Pierre Mendès France, 69676 Bron Cedex, France

e-mail : Jordan.navarro@univ-lyon2.fr

Daniel Ndiaye $(\triangle)$

Ifsttar-COSYS-LEPSIS

25 avenue François Mitterrand, 69675 Bron Cedex, France

e-mail : daniel.ndiaye@ifsttar.fr

Christophe Jallais $(\bowtie)$

Ifsttar-TS2-LESCOT

25 avenue François Mitterrand, 69675 Bron Cedex, France

e-mail : christophe.jallais@ifsttar.fr dispositif à l'intérieur du véhicule couplé ou non avec une manipulation manuelle. Toutefois, quelques études se sont également intéressées à l'impact de tâches distractives ayant seulement une composante cognitive. Les données montrent que l'efficacité d'une alerte peut dépendre de l'interaction entre sa modalité sensorielle et celle de la tâche distractive. Enfin, l'efficacité de ces systèmes peut également être impactée par l'interaction entre sa fiabilité et le fait d'être ou non distrait.

Cette revue soulève des questions de recherche qui devraient encore être approfondies en vue de garantir la sécurité du plus grand nombre d'usagers.

Mots clés distraction · alerte sonore · fiabilité · assistance à la conduite $\cdot$ attention

\begin{abstract}
This paper presents a review of the literature to shed light on the impact of distractions on the effectiveness of warning indicating a risk of collision.

The rear-end collisions account for a significant share of injury accidents. One of the major contributing factors to these collisions is drivers' inattention. To reduce the impact of this kind of collisions, driving assistance consisting in alerts have emerged. The effectiveness of these alerts has been evaluated in different situations (simulation, simulator and on road) in non-distracted and distracted drivers. In the latter case, the majority of distraction tasks used involves a diversion of eyes off the road to a device inside the vehicle, coupled or not with manual handling. However, few studies have also examined the impact of distractive tasks having only a cognitive component. The data show that the efficiency of an alert may depend on the interaction between its sensory modality and that of the distractive task. Finally, the effectiveness of these systems can also be affected by the interaction between its reliability and being or not distracted. This review raises research questions that should be further developed to ensure the safety of a larger number of users.
\end{abstract}

Keywords distraction - auditory warning - reliability . driving assistance $\cdot$ attention 


\section{Introduction}

Les collisions par l'arrière représenteraient environ $13 \%$ des accidents corporels sur les routes Européennes [1]. En 2013 et en France, selon l'Observatoire national interministériel de la sécurité routière [2], les collisions par l'arrière et collisions en chaîne auraient totalisé respectivement $4,9 \%$ et $1,1 \%$ de la mortalité routière. Elles auraient été également responsables respectivement de 7,9\% et 1,7\% des blessés graves de la route. En plus de ces taux non négligeables de mortalité et de blessures, ces collisions représentent un coût économique important pour la société et perturbent gravement la circulation.

Les facteurs contributifs à ce type de collisions peuvent être de nature environnementale, être liés au véhicule et/ou de nature humaine. Toutefois, il a été observé que la plupart de ces collisions se produisent pendant la journée (entre $9 \mathrm{~h} 30$ et $15 \mathrm{~h} \mathrm{30}$ ), dans de bonnes conditions météorologiques, sur des lignes droites et lorsque la surface de la chaussée est sèche et avec des vitesses modérées généralement inférieures à $60 \mathrm{~km} / \mathrm{h}$ [3-5]. Par conséquent, les conditions environnementales ne constitueraient un facteur contributif que dans 11 à $15 \%$ des collisions par l'arrière. Les problèmes techniques liés au véhicule, comme une faille des freins, expliqueraient quant à eux environ $10 \%$ de ces collisions [4,5]. Ainsi, c'est le facteur humain qui serait la principale cause des collisions par l'arrière, expliquant de $75 \%$ à $93 \%$ des cas selon les études [3-5]. L'adoption de distances inter-véhiculaires non sécuritaires est une cause majeure de ces collisions $[3,4]$. Or, selon le baromètre européen de la conduite responsable basé sur une enquête réalisée en janvier 2015 sur 9000 conducteurs européens, $65 \%$ des sondés reconnaissaient ne pas toujours respecter les distances de sécurité. Ce comportement est en progression dans l'ensemble des pays, notamment en France $(77 \%,+7$ points/2014) [6]. Un autre facteur contributif majeur à ces collisions est l'inattention des conducteurs qui peut se répercuter à la fois sur les distances inter-véhiculaires et sur les temps de réactions [3,4].

Afin de pallier ces risques de collisions, des systèmes d'alerte et d'évitement de collision ont été développés.

De manière synthétique, ces systèmes composés de capteurs, de radars et/ou caméras permettent de recueillir en temps réel la distance avec les éventuels obstacles détectés ainsi que la vitesse du véhicule afin de déterminer un risque de collision. Via un algorithme, le système détermine un seuil de déclenchement prenant en compte le temps avant collision calculé sur la base des indices précédents et des capacités de freinage du véhicule. Ces algorithmes peuvent également inclure des paramètres tels qu'un temps de réaction moyen, ou une force de décélération moyenne. Lorsque le seuil prédéfini est atteint, le système émet une alerte de type sonore, visuel et/ou tactile, afin d'avertir le conducteur qu'une réaction de sa part est requise pour éviter la collision (système d'avertissement). Lorsque le conducteur ne réagit pas les systèmes d'évitement peuvent prendre le contrôle du véhicule pour initier le freinage (système correctif). Il existe deux types de systèmes d'évitement : les systèmes urbains fonctionnant jusqu'à $30 \mathrm{~km} / \mathrm{h}$ et les systèmes interurbains agissant généralement à des vitesses comprises entre 50 et $80 \mathrm{~km} / \mathrm{h}$. Si ces systèmes ne permettent pas toujours d'éviter la collision, ils permettent en général de réduire la vitesse à l'impact (notamment en préchargeant les freins) et d'amoindrir ainsi les conséquences de la collision. Les consignes d'utilisation précisent en général que l'efficacité du système peut dépendre de plusieurs facteurs (par ex. vitesse, conditions routières...) et ils ne dédouanent pas d'une conduite sécuritaire et prudente.

Selon une étude récente, ces systèmes d'évitement de collisions pourraient réduire le nombre de collisions jusqu'à $38 \%$ [7]. L'efficacité des systèmes d'alerte dans leur capacité à éviter des collisions ou amoindrir leurs effets dépend de plusieurs facteurs liés à la fois au conducteur et à ses aptitudes ainsi qu'au système lui-même (fiabilité propre au système, moment de déclenchement de l'alerte, type d'alerte utilisée), facteurs qui interagissent les uns avec les autres.

Cette revue de la littérature s'intéresse à la fonction d'avertissement des systèmes et vise à offrir une synthèse des travaux ayant examiné l'efficacité de ces alertes selon l'état attentionnel des conducteurs. Nous présenterons dans un premier temps les données de la littérature concernant l'impact de ces alertes selon le type de tâches secondaires réalisées par les conducteurs, puis nous présenterons les données de la littérature s'intéressant à l'impact de la fiabilité de l'alerte toujours en lien avec la distraction.

\section{Efficacité des alertes anti-collision chez le conducteur distrait}

\section{Conduite et distraction}

Comme il a été évoqué précédemment, l'une des principales causes des collisions par l'arrière correspond aux défauts d'attention du conducteur. Ces défauts d'attention peuvent être dus à des baisses de vigilance ou des périodes de somnolence. Ils peuvent également être dus à des activités dites secondaires, non liées à la tâche principale de conduite et impliquant de détourner le regard de la route et/ou les mains du volant de façon fréquente (téléphoner, changer un $\mathrm{CD}$, manger...). La multi-activité au volant est devenue chose courante sur les routes. La législation française prévoit que « Tout conducteur doit se tenir constamment en 
état et en position d'exécuter commodément et sans délai toutes les manœuvres qui lui incombent. Ses possibilités de mouvement et son champ de vision ne doivent pas être réduits par le nombre ou la position des passagers, par les objets transportés ou par l'apposition d'objets non transparents sur les vitres » (article R412-6 ; décret $\mathrm{n}^{\circ}$ 2008-754 du 30 juillet 2008 - art. 15). De ce fait les activités telles que manger, fouiller dans la boîte à gants, ou encore regarder un écran non destiné à l'aide à la conduite peuvent être verbalisées. Depuis peu, la loi interdit également aux conducteurs de porter à l'oreille tout dispositif susceptible d'émettre du son (décret $n^{\circ}$ 2015-743 du 24 juin 2015 relatif à la lutte contre l'insécurité routière).

Mais outre ces activités distractives, notre attention peut également être attirée par une information saillante dans la scène routière que nous traiterons au détriment d'une autre information moins saillante mais essentielle pour la sécurité. Par ailleurs, l'organisation et les soucis de la vie quotidienne nous entraînent à penser à une multitude de choses, plutôt que de focaliser notre attention sur la route. Des données épidémiologiques ont mis en évidence que ce vagabondage de la pensée peut avoir des répercussions en termes de sécurité routière. Ainsi Galéra et al. [8] ont montré que parmi des conducteurs accidentés, ceux rapportant avoir été distraits par des pensées vagabondes au moment de l'accident avaient plus de chance d'être responsables de l'accident (odd ratio ajusté 2.12).

Toutes ces activités secondaires, qu'elles soient de caractère cognitif, perceptif (c'est-à-dire visuel, auditif) ou moteur, peuvent impliquer une importante réduction des ressources attentionnelles allouées à la tâche de conduite.

Parmi les activités entrant en compétition avec la tâche de conduite, ce sont celles de type visuo-motrices (c'est-à-dire écrire un texto au téléphone) impliquant de détourner le regard de la route et de lever les mains du volant qui ont été le plus souvent étudiées. En général, ces tâches sont associées à une dégradation dans le contrôle de la trajectoire latérale de la voie [9], une augmentation du nombre de regards hors de la route [10], une augmentation des temps de réaction au freinage du véhicule précédent [9] ainsi qu'une réduction de la distance inter-véhiculaire [11].

De façon intéressante, certains auteurs ont examiné l'effet différencié de tâches distractives visuelles et cognitives. Par exemple, Kaber et al. [10] ont montré une augmentation des regards hors de la route lors de la réalisation d'une tâche secondaire visuelle (simulant la demande visuelle lors de l'usage d'un système de navigation) alors que ces regards hors de la route étaient diminués lors de la réalisation d'une tâche secondaire cognitive (simulant la demande cognitive lors de l'écoute des instructions données par un système de navigation sans retour visuel). Par ailleurs, il a été montré que si une distraction visuelle (tâche de recherche visuelle sur un écran) pouvait altérer la perception et donc la réaction à des événements soudains et critiques, une distraction cognitive (compter à rebours de 3 en 3 à partir d'un nombre à 3 chiffres) impactaient l'anticipation des futures actions des autres conducteurs lors d'une tâche de suivi de véhicule (non-adaptation de la vitesse et des distances de sécurité) [12].

Si certains conducteurs peuvent adopter des stratégies de compensation en cas de distraction, comme une réduction de la vitesse [13] ou une augmentation de la distance inter-véhiculaire [10], ces mesures ne sont pas toujours efficaces en cas d'urgence. C'est donc dans ces conditions, que les systèmes d'alerte anti-collision peuvent être particulièrement utiles aux conducteurs distraits. Toutefois, s'ils ont été conçus pour remédier aux défauts d'attention des conducteurs, il n'est pas toujours évident de déterminer comment différents types de distraction des conducteurs influent sur leur efficacité.

\section{Alerte anti-collision, distraction visuelle et visuo-motrice}

L'impact de distractions induites par la réalisation de tâches visuelles ou visuo-motrices est facilement appréhendable du fait du détournement du regard de la scène routière vers un élément à l'intérieur ou à l'extérieur de l'habitacle. Dans ce cas, l'usage d'une alerte va permettre d'avertir le conducteur d'un risque potentiel et ainsi l'inciter à réorienter son regard et son attention sur la route.

Ainsi, en comparant les performances de conducteurs distraits à celles de conducteurs non-distraits plusieurs études sur simulateur ont mis en évidence l'intérêt de tels systèmes d'alerte. Lee et collaborateurs [14] ont observé un effet positif d'un système anti-collision combinant une alerte auditive et une alerte visuelle, pour les conducteurs non distraits ainsi que pour ceux distraits par une tâche visuelle. La tâche consistait à détecter le nombre 4 apparaissant sur un écran. Plus précisément, leurs résultats ont montré des marges de sécurité plus larges, des temps de réaction plus rapides et des décélérations plus progressives lorsque le système était disponible. En outre, le système a également eu un impact en réduisant le nombre de collisions ainsi que la vitesse à l'impact, ce bénéfice étant plus important pour les conducteurs distraits que pour les non distraits. Ho et Spence [15] ont également observé un effet positif d'une alerte auditive (temps de réactions et de réponses correctes) dans la détection d'une possible collision que cela soit chez des participants non distraits ou distraits. Les participants étaient distraits par une tâche visuelle consistant à discriminer la couleur de LED situées à leur droite ou à leur gauche. Quant à Kramer et al. [16], ils ont obtenu des résultats similaires en utilisant une tâche distractive visuo-motrice (composer un code de 7 chiffres 
sur un écran tactile) et un signal sonore et visuel. De plus, ils ont trouvé un bénéfice en termes de diminution du nombre des collisions que cela soit pour les conducteurs distraits ou non-distraits.

D'autres études sur simulateur se sont attachées à montrer les effets bénéfiques des systèmes d'avertissement anticollision mais uniquement sur des groupes de participants distraits. Par exemple, les résultats de Maltz et Shinar [17] ont montré que les participants distraits par une tâche visuelle consistant à détecter des changements sur des cibles en périphérie et qui conduisaient avec l'aide d'un signal avertisseur auditif, passaient plus de temps avec des marges de sécurité plus sûres (TIV $>1$ seconde) et moins avec des marges moins sûres (TIV $<1$ seconde) que les participants qui ne disposaient pas du système. De même, Chun et al. [18] ont trouvé que la présence d'un signal tactile aidait les participants distraits par une tâche visuo-motrice (composition d'un code à 7 chiffres sur un écran) à réagir plus rapidement aux décélérations brusques du véhicule précédent et à éviter plus de collisions que lorsque le signal n'était pas disponible.

Enfin, Kiefer et al. [19] ont montré le bénéfice d'un système anti-collision composé d'un signal auditif et visuel dans des conditions de conduite plus réalistes (sur piste). Cependant, et de façon intéressante leurs résultats dépendaient du type de tâche secondaire réalisée par les participants. En effet, le système était efficace lorsque les participants réalisaient une tâche de distraction visuomotrice (consistant à composer une série de chiffres sur un téléphone portable fixé dans l'habitacle). En revanche, le système n'a pas montré d'effets positifs lorsque les participants réalisaient une tâche de distraction cognitive (déterminer si une phrase énoncée oralement était vraie ou fausse) n'impliquant pas de détourner le regard de la route.

L'efficacité d'une alerte pourrait également dépendre de la concordance possible entre sa modalité et celle(s) impliquée(s) par la tâche secondaire. En effet, les données de la littérature montrent que la probabilité d'interférence entre deux tâches est plus importante si ces tâches ont en commun la même modalité [20]. En accord avec cette théorie, Mohebbi et Gray [21] ont observé que lorsque les conducteurs sont engagés dans une tâche secondaire impliquant la modalité auditive (conversation téléphonique), une alerte sonore signalant un risque de collision était moins efficace qu'une alerte tactile. Or on notera que dans les études présentées ci-dessus, si les tâches secondaires employées étaient visuelles ou visuo-motrices les alertes employées étaient sonore, sonore et visuelle, ou tactile, assurant ainsi une faible interférence de la tâche secondaire sur le traitement de l'alerte. Il pourrait donc être intéressant de poursuivre l'évaluation de l'efficacité de ce type d'alerte en manipulant les modalités de la tâche secondaire.

\section{Alerte anti-collision et distraction cognitive}

Depuis l'étude de Kiefer et al. [19] qui suggère des effets différents sur l'efficacité d'une alerte pour les distractions de type cognitif et celles de type visuel ou visuo-motrice, relativement peu de travaux ont cherché à estimer l'efficacité des systèmes d'alertes lors de réalisation de tâches secondaires de nature purement cognitive. Ces tâches ne nécessitent pas d'ôter les mains du volant ou de détourner le regard de la route mais, pour autant, elles détournent l'attention des conducteurs de la route vers leurs propres pensées.

Abe et al. [22] sur simulateur de conduite ont montré des bénéfices d'un signal auditif uniquement chez les conducteurs distraits par une tâche nécessitant à la fois des ressources visuo-motrices et cognitives (additionner 4 chiffres présentés sur un écran et entrer le résultat). Cependant, les auteurs ont suggéré que ce résultat pourrait être expliqué par des conditions de trafic intense où la haute fréquence de déclenchement du signal pourrait être perçue comme inutile. Les données subjectives (via une échelle en 11 points permettant d'évaluer la nécessité des alertes auditives) ont confirmé cette hypothèse, montrant que les conducteurs non distraits évaluaient les signaux avertisseurs plus négativement.

Au sein de notre laboratoire, nous avons réalisé une série d'études visant à examiner l'effet d'une alerte anti-collision en conduite simulée chez des conducteurs réalisant une tâche secondaire cognitive [23-26]. Cette tâche consistait pour le participant à écouter trois mots sans lien direct les uns avec les autres et à trouver un quatrième mot lié à chacun des mots présentés, que cela soit par le biais d'une expression, d'une synonymie ou d'un lien sémantique (Exemple : «Crayon - Rien - Charbon »-réponse possible " Mine » pour « mine de crayon », " mine de rien » et «mine de charbon »). Un autre triplet de mots était proposé au participant dès qu'il donnait une réponse ou après une minute sans réponse. La tâche principale des participants était de suivre une moto et de réagir en décélérant dès que le feu stop de la moto s'allumait. Dans les 2 premières études $[24,25]$, nous avons recueilli des données comportementales (temps de réponse) et électrophysiologiques à savoir l'activité cérébrale via un électroencéphalogramme et à l'analyse des potentiels évoqués associés. Un potentiel évoqué correspond à la réponse électrique du cerveau à une stimulation. Il se compose d'une succession d'ondes ou composantes qui sont définies par leur polarité ainsi que la latence et l'amplitude de leur pic. Schématiquement, ces différentes composantes correspondent aux différentes étapes du traitement de l'information. Les modulations observées en termes d'amplitude et de latence des différentes composantes apportent des indications quant aux 
étapes du traitement de l'information qui sont impactées par telle ou telle condition expérimentale. Ainsi nous avons pu observer l'impact d'une alerte auditive et/ou de notre tâche de distraction cognitive sur les potentiels évoqués par l'allumage du feu stop de la moto précédente. Les résultats comportementaux ont montré que les participants étaient plus rapides à répondre à l'allumage du feu de la moto lorsqu'ils bénéficiaient de l'alerte sonore qu'ils soient distraits ou non. Au niveau électrophysiologique, cet effet d'alerte s'est traduit par une amélioration des processus d'anticipation et par une accélération du traitement du feu de la moto. Toutefois, un fort impact négatif de la tâche secondaire a été observé au niveau comportemental comme au niveau électrophysiologique avec et sans alerte. Ce résultat suggère que la présence de l'alerte n'était pas suffisante pour compenser complètement l'effet négatif de la tâche secondaire.

Sachant que la distraction cognitive altère les comportements d'anticipation (adaptation des vitesses et des distances de sécurité) [12] et que l'alerte semble agir au niveau des processus d'anticipation [25], on aurait pu s'attendre à ce que l'alerte compense les effets de la distraction. Deux explications non exclusives peuvent expliquer cette absence de résultat. D'une part, comme nous l'avons évoqué dans le paragraphe précédent, si la tâche secondaire et l'alerte partagent la même modalité sensorielle, il est possible que les traitements des deux informations interfèrent diminuant ainsi l'efficacité de l'alerte. Or notre tâche secondaire outre l'effort cognitif qu'elle demandait, utilisait le canal auditif dans la phase de présentation des triplets de mots. D'autre part, la complexité de notre tâche cognitive pourrait elle aussi expliquer cette absence de résultats. En effet, il est connu que la quantité de ressources attentionnelles disponibles est considérablement réduite par la présence d'une tâche secondaire [27, 20]. Plus les tâches secondaires sont exigeantes sur le plan cognitif, plus les ressources attentionnelles nécessaires pour traiter les deux tâches en même temps sont importantes.

Dans ce contexte, il est possible qu'un individu ne dispose pas d'assez de ressources pour traiter efficacement une alerte et en tirer profit. Afin de tester cette hypothèse, nous avons réalisé une troisième étude sur simulateur, dans laquelle nous avons fait varier le niveau de difficulté de la tâche secondaire [26]. Nous avons de nouveau utilisé la tâche des triplets de mots mais en faisant varier le niveau de difficulté selon les participants. Ainsi un groupe de participants étaient soumis à une liste de triplets dont la solution était évidente et un deuxième groupe était soumis à une liste de triplets dont la solution était difficile à trouver. Dans cette étude, seules des mesures comportementales ont été enregistrées. Les résultats ont montré que lorsque l'alerte sonore était disponible, les temps de réponse étaient plus courts que dans la condition sans alerte. Cet effet bénéfique de l'alerte a été également observé pour les participants distraits par la tâche secondaire de faible difficulté mais pas pour ceux distraits par une tâche secondaire de difficulté élevée. Ce résultat suggère donc que le bénéfice potentiel d'une alerte dépend, au moins en partie, des ressources attentionnelles disponibles.

Des travaux supplémentaires semblent nécessaires afin de mieux cerner l'impact des tâches secondaires sur l'efficacité des alertes en dissociant leurs modalités. Par ailleurs, l'usage d'alertes bimodales pourrait être une solution pertinente pour assurer leur efficacité dans une plus large proportion de situations [voir 28, 15].

\section{Efficacité vs fiabilité des systèmes d'alerte anti-collision}

L'efficacité des systèmes d'avertissement ou d'évitement des collisions dépend de plusieurs paramètres dont la fiabilité effective du système et de l'acceptabilité par l'usager. Selon les technologies employées les systèmes sont susceptibles de présenter différentes limites. Outre les limites concernant les fenêtres de vitesses sur lesquelles les systèmes peuvent être utilisés, certains capteurs peuvent ne détecter que des surfaces métalliques, avoir un fonctionnement limité dans certaines conditions météorologiques... Par ailleurs, selon le seuil de déclenchement retenu, le système peut produire des fausses alarmes et/ou des absences de détection des événements critiques. Les fausses alarmes se réfèrent à des situations dans lesquelles un signal avertisseur est déclenché en l'absence de risque de collision. Les absences de détection se produisent lorsqu'un signal avertisseur n'est pas déclenché bien que la situation l'exigerait. On note également que dans les virages, ils peuvent signaler de « faux » obstacles notamment en détectant un véhicule sur la voie de circulation inverse ou ne pas détecter à temps un véhicule devant du fait de la courbure du virage.

$\mathrm{Du}$ point de vue de l'acceptabilité, nous noterons qu'un système trop sensible, se déclenchant de façon non appropriée (fausses alarmes) pourrait être mal apprécié des usagers et irriter le conducteur. Si l'information fournie est trop souvent non pertinente, elle pourrait être négligée même lorsqu'elle est pertinente [29] ou même pousser l'usager à désactiver le système.

En plus des conséquences sur l'acceptabilité du système, un nombre trop élevé de fausses alarmes peut générer des changements sur le comportement des conducteurs. Ainsi, les fausses alarmes récurrentes semblent allonger le temps de réaction au freinage [30] et induire des réponses de décélération non nécessaires [17]. 
Les absences de détections de possibles collisions peuvent également affecter l'acceptation du système et la performance des conducteurs. En effet, un système trop sélectif pouvant manquer des événements critiques pourrait être perçu comme inutile et être rejeté par le conducteur [31, 32]. De plus, si le système ne fonctionne pas correctement, le temps de réaction nécessaire en situation de collision peut être allongé considérablement, et pourrait même être plus long que lorsque le véhicule ne dispose pas du système [30].

Les résultats obtenus soit par des études réalisées sur route réelle [33], soit par de la modélisation à partir de données de l'accidentologie [34] ont montré une faible précision du système (entre 32 et $56 \%$ ) et un pourcentage très élevé de fausses alarmes (environ $60 \%$ ). Toutefois et bien qu'il reste difficile d'avoir des informations précises sur la fiabilité des systèmes, il semble que les technologies ont très nettement progressé ces dernières années.

Quoi qu'il en soit, un autre point critique concerne l'interaction possible entre la fiabilité effective d'un système et certaines caractéristiques propres aux conducteurs telles que son âge, son expérience de conduite [35], ou encore son état attentionnel. Par exemple, une alerte déclenchée trop tôt pourrait être considérée comme une nuisance pour un conducteur non distrait, alors qu'elle pourrait être efficace pour un conducteur distrait. Ainsi, Abe et al. [30] ont découvert que les conducteurs évaluaient les alertes comme moins inutiles quand ils étaient distraits par une tâche visuelle secondaire que quand ils étaient sans distraction. Il semble donc que les conducteurs distraits pourraient compenser leur manque de ressources attentionnelles en se reposant plus sur le système. Maltz et Shinar [17] n'ont pas trouvé de différences chez les conducteurs distraits en ce qui concerne le pourcentage de temps passé dans une zone de sécurité ou de danger selon la fiabilité du système (élevé, moyen et faible fiabilité ou 1,4 et 8 erreurs par minute, respectivement). Néanmoins, l'analyse de la performance des conducteurs lorsque le système n'a pas réussi à fournir une alerte a montré un taux de réponses de décélération dans la zone de danger plus élevé pour le groupe bénéficiant d'un système avec une fiabilité faible par rapport aux groupes bénéficiant d'un système à fiabilité haute ou moyenne et par rapport au groupe contrôle. Ce résultat pourrait suggérer que même lorsqu'ils sont distraits, les conducteurs peuvent adopter un comportement sécuritaire lorsque le système ne détecte que rarement les dangers. Cependant, lorsque le système est très fiable, les conducteurs pourraient s'appuyer de façon excessive sur le système, et ne compenseraient pas l'erreur du système quand elle intervient. Comme dans l'étude de Malt et Shinar [17], Bueno et al. [24] ont examiné l'effet du niveau de fiabilité d'une alerte signalant un risque de collision (fiable à $100 \%$ ou $70 \%$ ) chez les conducteurs non-distraits et distraits par une tâche secondaire cognitive. Les données comportementales ont montré que les alertes plus ou moins fiables réduisaient uniquement les temps de réponses des conducteurs non-distraits. Toutefois, l'analyse de l'activité électrique cérébrale via les potentiels évoqués suggère : 1. qu'en simple tâche l'alerte fiable à $100 \%$ favorise l'attente de la cible; et 2 . de façon plus surprenante, qu'en double tâche, l'alerte fiable à $70 \%$ réduirait le temps requis pour le traitement de la cible et la prise de décision la concernant. Ainsi et parallèlement à l'étude de Maltz et Shinar [17], mais avec toute la prudence nécessaire, on pourrait spéculer que les systèmes moins fiables peuvent être plus efficaces que des systèmes plus fiables dans des situations de double tâche. Néanmoins, des recherches supplémentaires sont nécessaires pour faire la lumière sur ces phénomènes.

\section{Conclusion}

L'efficacité de la fonction d'avertissement des systèmes anti-collision repose sur de nombreux facteurs. Dans cette revue, deux facteurs ont été discutés à savoir l'état attentionnel des conducteurs et la fiabilité des alertes.

Les travaux présentés participent à mettre en évidence que l'efficacité de la fonction d'alerte signalant un risque de collision peut être modulée par l'état attentionnel des conducteurs. Sachant que ces systèmes sont conçus pour pallier les erreurs humaines et notamment les défauts d'attention des conducteurs, ces résultats renforcent l'idée que l'efficacité de ces systèmes d'assistance à la conduite devrait être évaluée dans différents contextes de distraction des conducteurs. En effet, les études montrent que leur efficacité peut varier en fonction de leur modalité sensorielle et du type de tâches distractives (visuelles, visuo-motrices, cognitives) réalisées par les conducteurs. Sur la base de ces travaux, il semble également utile d'évaluer l'efficacité de ces systèmes en prenant en considération les capacités, en particulier attentionnelles, des usagers.

Cette revue aborde également la question de l'interaction entre la fiabilité des systèmes et l'état attentionnel des conducteurs. Un approfondissement de cette question nous semble essentiel afin de mieux cerner l'impact de ces interactions en termes d'acceptabilité et de leurs répercussions en termes de sécurité routière en vue de garantir la sécurité du plus grand nombre.

\section{Remerciements}

Les travaux de recherche présentés dans ce papier ont bénéficié du soutien de la Commission Européenne (7th framework program FP7/2007-2013) dans le cadre du projet ADAPTATION (grant agreement $n^{\circ} 238833$ ) ainsi 
que du soutien de la Fondation pour la sécurité routière (2012/MP/05).

\section{Bibliographie}

1. Van Kampen B (2003) Case study: rear end or chain accidents. The Netherlands: SWOV Institute for Road Safety Research.

2. Observatoire national interministériel de la sécurité routière (2013) Les accidents corporels de la circulation. Recueil de données brutes « Documents de travail », $88 \mathrm{p}$.

3. Dingus TA, Klauer SG, Neale VL et al. (2006) The 100-Car Naturalistic Driving Study, Phase II-Results of the 100-Car Field Experiment, Washington, DC: National Highway Traffic Safety Administration.

4. Knipling RR, Wang JS, Yin HM (1993) Rear-end Crashes: Problem size assessment and statistical description. Washington, DC: National Highway Traffic Safety Administration.

5. Vogel L, Bester CJ (2005) A relationship between accident types and causes. Paper presented at the 24th Southern African Transport Conference, South Africa.

6. http://www.vinci-autoroutes.com/fr/system/files/pdf/2015/02/ barometre conduite responsable_-_edition_2015.pdf

7. Fildes B, Keall M, Bos N, Lie A, Page Y, Pastor C, Pennisi L, Rizzi M, Thomas P, Tingvall C (2015) Effectiveness of low speed autonomous emergency braking in real-world rear-end crashes. Acc Anal Prev 81: 24-29.

8. Galéra C, Orriols L, M'Bailara K et al. (2012) Mind wandering and driving: responsibility case-control study. BMJ 345: e8105.

9. Drews FA, Yazdani H, Godfrey CN et al. (2009) Text Messaging During Simulated Driving. Hum Factors 51(5): 762-770.

10. Kaber DB, Liang Y, Zhang Y et al. (2012) Driver performance effects of simultaneous visual and cognitive distraction and adaptation behavior. Transport Res F-Traf 15(5): 491-501.

11. Jamson AH, Merat N (2005) Surrogate in-vehicle information systems and driver behaviour: Effects of visual and cognitive load in simulated rural driving. Transport Res F-Traf 8(2): 79-96.

12. Muhrer E, Vollrath M (2011). The effect of visual and cognitive distraction on driver's anticipation in a simulated car following scenario. Transportation Research Part F: Traffic Psycholgy and Behavior 14(6): 555-566.

13. Engström J, Johansson E, Östlund J (2005) Effects of visual and cognitive load in real and simulated motorway driving. Transport Res F-Traf 8(2): 97-120.

14. Lee JD, McGehee DV, Brown TL, Reyes ML (2002) Collision Warning Timing, Driver Distraction, and Driver Response to Imminent Rear-End Collisions in a High-Fidelity Driving Simulator. Hum Factors 44(2): 314-334.

15. Ho C, Spence C (2009) Using peripersonal warning signals to orient a driver's gaze. Hum Factors 51(4): 539-556.

16. Kramer AF, Cassavaugh N, Horrey WJ et al. (2007) Influence of Age and Proximity Warning Devices on Collision Avoidance in Simulated Driving. Hum Factors 49(5): 935-949.

17. Maltz M, Shinar D (2004) Imperfect in-vehicle collision avoidance warning systems can aid drivers. Hum Factors 46(2): 357-366.
18. Chun J, Han SH, Park G, Seo J et al. (2012) Evaluation of vibrotactile feedback for forward collision warning on the steering wheel and seatbelt. Int J Ind Ergonom 42(5): 443-448.

19. Kiefer R, LeBlanc D, Palmer M et al. (1999) Development and validation of functional definitions and evaluation procedures for collision warning/avoidance systems. Washington, DC: National Highway Traffic Safety Administration.

20. Wickens C (1980) The structure of attentional resources. In: Nickerson R, editor. Attention and performance. Hillsdale, NJ: Erlbaum, p. 239-257.

21. Mohebbi R, Gray R, Tan HZ (2009) Driver Reaction Time to Tactile and Auditory Rear-End Collision Warnings While Talking on a Cell Phone. Hum Factors 51(1): 102-110.

22. Abe G Itoh M \& Yamamura T (2011) Visual distraction when driving and its influence on driver response to a forward collision warning system. Paper presented at the SICE Annual Conference, Tokyo, Japan.

23. Bueno-Garcia M (2013) Impact d'un système anticollision sur le traitement de l'information et le comportement du conducteur. Thèse de Doctorat en Neurosciences, Université Bordeaux 2 et Ifsttar/TS2/Lescot, $131 \mathrm{p}$.

24. Bueno M, Fabrigoule C, Deleurence P, et al.. (2012) Analysis of a surrogate forward collision warning system from an electroencephalographic perspective. Brain Res 1470: 69-79.

25. Bueno M, Fort A, François et al. (2013) Effectiveness of a Forward Collision Warning System in simple and in dual task from an electrophysiological perspective. Neurosci Lett 541: 219-223.

26. Bueno M, Fabrigoule C, Ndiaye D, Fort A (2014) Behavioural adaptation and effectiveness of a Forward Collision Warning System according to a secondary cognitive task. Transport Res F-Traf 24: 158-168.

27. Baddeley AD (1996) Exploring the central executive'. The Quarterly Journal of Experimental Psychology 49A(1): 5-28.

28. Campbell JL, Richard CM, Brown JL, McCallum M. (2007). Crash Warning System Interfaces: Human Factors Insights and Lessons Learned. Final Report Seattle: Battelle Center for Human Performance and Safety.

29. Lerner ND, Dekker DK, Steinberg GV, Huey RW (1996) Inappropriate Alarm Rates and Driver Annoyance. Washington, DC: National Highway Traffic Safety Administration.

30. Abe G, Itoh M, Tanaka K (2002) Dynamics of drivers' trust in warning systems. Paper presented at the 15 th IFAC World Congress, Barcelona, Spain.

31. Bliss JP, Acton SA (2003) Alarm mistrust in automobiles: how collision alarm reliability affects driving. Appl Ergon 34(6): 499509.

32. Lees MN, Lee JD (2007) The influence of distraction and driving context on driver response to imperfect collision warning systems. Ergonomics 50(8): 1264-1286.

33. Najm WG, Stearns MD, Howarth $\mathrm{H}$ et al. (2006) Evaluation of an automotive rear end collision avoidance system. Cambridge: US Dept of Transportation.

34. McLaughlin SB, Hankey JM, Dingus TA, Klauer SG (2009) Development of an FCW Algorithm Evaluation Methodology With Evaluation of Three Alert Algorithms. Virginia: VTTI.

35. Lees M (2010) Context conditions drivers' disposition towards alarms. Philosophy degree in Industrial Engineering, University of Iowa, Iowa. 